\title{
A Hybrid Dynamic QRDM and ZF Detection Algorithm for MIMO-OFDM Systems
}

\author{
Weizhi You, Lilin $\mathrm{Yi}^{*}$ and Weisheng $\mathrm{Hu}$ \\ State Key Lab of Advanced Optical Communication Systems and Networks \\ Dept. Electronic Engineering, Shanghai Jiao Tong University \\ 800 Dongchuan Rd, Shanghai, 200240, China. \\ *E-mail: lilinyi@sjtu.edu.cn
}

\begin{abstract}
An effective signal detection algorithm with low complexity is presented for MIMO-OFDM systems. The proposed technique combines a dynamic $Q R$ decomposition based M-Algorithm (QRDM) and Zero-Forcing (ZF) detection. In the systems, QRDM is executed for the first $N_{T}-\left[N_{T} / 3\right\rceil+1$ layers,

$\mathrm{ZF}$ detection is used for the last $\left\lceil N_{T} / 3\right\rceil-1$ layers, where $N_{T}$ means the number of transmitter antennas. Compared with the conventional QRDM algorithm, this approach is simple and has low complexity because not all branches are searched. Furthermore, the performance of this algorithm is similar to that of the conventional QRDM algorithm. The receiver detection simulation is based on five paths Rayleigh fading environment without channel coding, assuming the channel matrix $H$ is perfectly known by the detector. From simulation results, the complexity of QRDM-ZF detection algorithm for 4 transmitter antennas and 4 receiver antennas systems with quadra-binaryshift-keying (QBSK) modulation is reduced by $29.41 \%$ on average and it is reduced by $30.08 \%$ on average for 16 -quadraamplitude-modulation (QAM). The performance degradation is about $2 \mathrm{~dB}$ at $B E R=10^{-3}$. The proposed hybrid QRDM-ZF detection can be used for a MIMO-OFDM receiver requiring not very high performance but needing very low complexity.
\end{abstract}

Keywords-MIMO-OFDM;QR-decomposition;Zero-Forcing; QRDM algorithm; QRDM-ZF algorithm .

\section{INTRODUCTION}

The multiple-input multiple- output (MIMO) architecture provides significant capacity gain in wireless channels. And orthogonal frequency division multiple (OFDM) has become a popular technique for transmission of high data rate wireless communication because of its inherent error susceptibility in a multipath environment. OFDM based on MIMO is a key technique for the next generation wireless communication. In the MIMO-OFDM system, a signal detection algorithm with a low complexity and high BER performance is a troublesome subject. Therefore, lots of signal detection algorithms have been proposed [1]-[4]. There are QR [5], Zero-Forcing (ZF) [6] and a dynamic QR decomposition based M-Algorithm (QRDM) [7]. Among those detection algorithms, the QRDM algorithm has very high performance. Unfortunately, since this algorithm is a tree search process, the detection complexity will be highly increased when the number $M$ increases.

In this paper, a simple and efficient detection technique based on hybrid QRDM and ZF is presented for MIMO-OFDM systems, for the first time. A new parameter $\left[N_{T} / 3\right\rceil$ has been adopted, where $N_{T}$ means the number of transmitter antennas. In the first part of $N_{T}-\left\lceil N_{T} / 3\right\rceil+1$ layers, QRDM algorithm is executed.
Then ZF detection is used for the second part including last $\left\lceil N_{T} / 3\right\rceil-1$ layers. Before detection, the channel matrix $H$ has been sorted as the column norm from the minimum to the maximum. By this way, the performance of the first detected signals has enhanced greatly comparing with the conventional QRDM algorithm. The reason why ZF detection is used is that the performance of the first part detected layers is the best in all layers. When the first signals are reserved, its interference to other layers may be cancelled, and then the ZF algorithm will be much more reliable in the second part detection. Form simulation results, compared with QRDM algorithm, the QRDM-ZF detection performance is decreased $2 \mathrm{~dB}$ at $\mathrm{BER}=10^{-3}$. However, its complexity has decreased significantly.

The rest of this paper is organized as follows. The MIMO-OFDM systems model is described In Section II. In Section III, several conventional MIMO detection algorithms are briefly introduced. In section IV, a low complexity hybrid QRDM and linear ZF detection is proposed. The simulation results are presented in section $\mathrm{V}$ and Section VI is conclusions.

\section{SYSYEM MODEL}

\section{A. MIMO-OFDM Systems}

Consider a communication system with $N_{T}$ transmitter antennas and $N_{R}$ receiver antennas $\left(N_{R} \geqslant N_{T}\right)$, denoted by $\left(N_{T}, N_{R}\right)$. Fig.1 (a) is the structure of a MIMO-OFDM transmitter. At the transmitter the input information is demultiplexed and coded to generate $N_{T}$ symbol streams by serial-parallel converting. The symbol streams are lunched into the constellation mapping, the Inverse Fast Fourier Transform (IFFT) modulators and added cyclic prefix (CP). Finally, the number of $N_{T}$ OFDM signals is transmitted by every transmitter antenna. Fig. 1(b) shows the block structure of a MIMO-OFDM receiver. Each receiver antenna receives signals from all transmit antennas.

After the cyclic prefix is removed, every received signal passes through a Fast Fourier Transform (FTT) block for demodulation. The receiver signal after demodulation, at receiver antennas can be given by

$$
y=H x+n
$$

Where $H$ is the complex-valued $N_{R} \times N_{T}$ matrix of quasi-static flat Rayleigh fading channel, y is a $N_{R} \times 1$ vector of received signals, $\mathrm{x}$ is the transmitted signals with $N_{T} \times 1$ vector, and $\mathrm{n}$ is $N_{R} \times 1$ additive noise white Gaussian (ANWG) vector. Let's denote $y=\left[y_{1}, \ldots, y_{N_{R}}\right]^{T}, x=\left[x_{1}, \ldots, x_{N_{T}}\right]^{T} n=\left[n_{1}, \ldots, n_{N_{R}}\right]^{T}$, and

$$
\left.H=\left(h_{i, j}\right)_{(1 \leqslant i \leqslant N R}, 1 \leqslant j \leqslant N T\right)
$$

This work was supported by 973 Program (2012CB315602, 2010CB328204-5), Nature Science Foundation China (61007041, 61090393, 61132004 and 60825103), 863 Program, Program of Shanghai Subject Chief Scientist (09XD1402200), Program of Shanghai Chen Guang Scholar (11CG11) and Program of Excellent PhD in China (201155). 
Where $h_{i, j}$ is a fading channel impulse response from transmitter antenna $j$ to receiver antenna $i$.

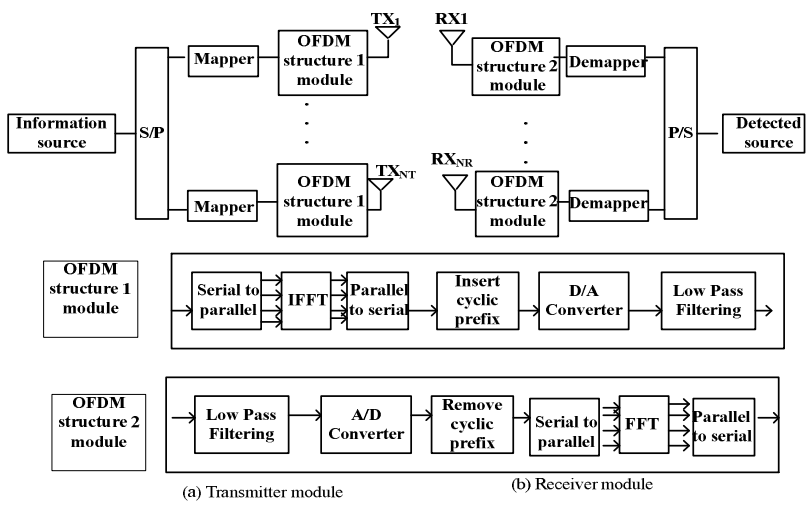

Figure. 1 The structure of MIMO-OFDM system

\section{B. Model Assumptions}

Additionally, in order to detect transmit signals, we make some assumptions:

(A1) The quasi-static flat Rayleigh fading channel matrix $H$ is perfectly known by the detector;

(A2) $n$ is zero mean, circularly symmetric complex-valued Gaussian with $E\left(n n^{H}\right)=\sigma_{n}^{2} I_{N_{R}}$;

(A3) $x$ is zero mean and with $E\left(x x^{H}\right)=I_{N_{T}}$;

(A4) $x$ and $n$ are independent of each other.

\section{Signal Detection AlgORITHM}

Because MIMO-OFDM can be seen as a flat MIMO system, and there are several conventional MIMO detection algorithms. Some of them can be directly applied to MIMOOFDM system.

\section{A. Linear ZF Receiver}

In a Zero-Forcing (ZF) receiver, the receiver vector $\mathrm{y}$ is multiplied by a filter matrix $\mathrm{G}$.

$$
G=\left(H^{H} H\right)^{-1} H^{H}
$$

Get the estimation value of transmitted signals by

$$
\tilde{x}=G y
$$

Where $x$ is the output of ZF.

\section{B. QR Receiver}

The QR decomposition of the channel matrix $H$ was introduced in [5] as another method to decode MIMO-OFDM systems. The channel matrix $H$ can be decomposed by $H=Q R$ using the $N_{R} \times N_{T}$ unitary matrix $Q$ and the $N_{T} \times N_{T}$ upper triangular matrix $R$. So the model (1) can be amended as

$$
\begin{aligned}
& y=Q R x+n \\
& Q^{H} y=R x+Q^{H} n \\
& \sim \\
& y=R x+\tilde{n}
\end{aligned}
$$

Where $\tilde{y}=Q^{H} y, \tilde{n}=Q^{H} n$.The statistical properties of the noise term $Q^{H} n$ remain unchanged.

The (5) can be written as below

$$
\left(\begin{array}{l}
\tilde{y_{1}} \\
\tilde{y_{2}} \\
\cdot \\
\cdot \\
\cdot \tilde{y_{N_{T}}}
\end{array}\right)=\left(\begin{array}{cccc}
r_{1,1} & r_{1,2} & \cdots & r_{1, N_{T}} \\
0 & r_{2,2} & \ldots & r_{2, N_{T}} \\
0 & 0 & \ldots & r_{3, N_{T}} \\
\cdot & \cdot & \cdots & \cdot \\
\cdot & \cdot & \cdots & \cdot \\
\cdot & \cdot & \cdots & \cdot \\
0 & 0 & \ldots & r_{N_{T} N_{T}}
\end{array}\right)\left(\begin{array}{l}
x_{1} \\
x_{2} \\
\cdot \\
\cdot \\
\cdot \\
x_{N_{T}}
\end{array}\right)+\left(\begin{array}{l}
\sim \\
n_{1} \\
\tilde{n_{2}} \\
\cdot \\
\cdot \\
\tilde{n_{N_{T}}}
\end{array}\right)
$$

The element $N_{T}$ of vector $y$ is

$$
\tilde{y}_{N_{T}}=r_{N_{T}, N_{T}} \cdot x_{N_{T}}+\tilde{n}_{N_{T}}
$$

Then, the decision statistic (7) can be used to estimate $\tilde{x}_{N_{T}}$, it is

$$
\tilde{x}_{N_{T}}=\text { Quant }\left[\frac{\tilde{y_{N_{T}}}}{r_{N_{T} N_{T}}}\right]
$$

Where Quant [] is the quantization operation.

If the decision of $N_{T}$ layer is correct, thus its interference term can be subtracted from the $N_{T}-1$ layer signal $\tilde{y}_{N_{T}-1}$ when $\tilde{x}_{N_{T}-1}$ is estimated. The process of QR detecting can be described by using the following recursive algorithm,

$$
\tilde{x}_{i}=\frac{1}{r_{i, i}}\left(\tilde{y}_{i}-\sum_{j=i+1}^{N_{T}} r_{i, j} \tilde{x}_{j}\right), i=N_{T}, N_{T}-1, N_{T}-2, \ldots 1
$$

If the ith layer is detected successively and wrong, those wrong decisions will affect the subsequent decisions.

\section{QRDM Receiver}

QRDM algorithm is proposed for recovering the data streams in MIMO-OFDM systems [7]. $M$ represents the number of branches that are kept in every layer. $M \in$ $P=\{1,2, \ldots, S\}$, where $P$ is the points set and $S$ is the total points on the modulation constellation. For example, if 16QAM is used, then $M$ can choose the value form 1 to 16 and $S$ is equal to 16. In order to illustrate the process of QRDM detection, effective number of branches should be denoted as $C_{i}$, where $i=1,2, \ldots, N_{T}$. Algorithm:

Step1: Determine the number of branches should be calculated in ith layer;

(a) if $\mathrm{i}=1$, then $C_{1}=S$;

(b) if $\mathrm{i}>1$, then $C_{\mathrm{i}}=S C_{\mathrm{i}-1}$.

Step 2: Calculate the expansion weight metrics of each branch in ith layer by (10);

$$
a_{i}(d)=\sum_{k=N_{T}+1-i}^{N_{T}}\left(\tilde{y}_{k}-\sum_{l=k}^{N_{T}} r_{k, l} x_{l}\right)^{2}, 1 \leq d \leq C
$$

Step 3: According to the value of $a_{i}(d)$, it need sort $a_{i}(d)$ from maximum to minimum;

(a) $i<N_{T}$, (I) if $C_{\mathrm{i}} \leqslant M$, then $\mathrm{i}=\mathrm{i}+1$, go to step 1 .

(II) if $C_{\mathrm{i}}>M$, then $M$ branches should be reserved and $C_{\mathrm{i}}=M, i=i+1$; go to step 1 .

(b) $i=N_{T}$, the algorithm is end and the points $x$ reserved in the smallest weight metrics of the branch is the output of QRDM algorithm.

For example, when $N_{T}=N_{R}=4$, QPSK modulation, the tree structure of QRDM algorithm is shown in Fig.2 (a). 
When $M=1$, the BER effective of QRDM receiver is similar to $\mathrm{QR}$ receiver. When $M=S$ (the total points on the modulation constellation), the BER effective of QRD-M receiver is similar to Maximum likelihood (ML) receiver.

\section{A HYBRID QRDM AND ZF DETECTION AlGORITHM} A. Algorithm Development

To the above MIMO-OFDM system model, a new detection scheme for MIMO channel matrix at every subcarrier is proposed. From [8], we can know when the first $N_{T}-\left\lceil N_{T} / 3\right\rceil+1$ layers are detected by QRDM algorithm and the last $\left[N_{T} / 3\right]-1$ layers are detected by $\mathrm{ZF}$ algorithm, the combined QRDM-ZF is reasonable, considering the compromise between the performance and the complexity of the proposed algorithm. The method consists of two parts. The first part is to apply the QRDM detection for obtaining the estimation of transmitting signals $\left[\tilde{x}_{\left\lceil N_{T} / 3\right\rceil}, \ldots, \tilde{x}_{N_{T-1}}, \tilde{x}_{N_{T}}\right]$, where $\left\lceil N_{T} / 3\right\rceil$ represent the minimum integer number that is bigger than $\mathrm{N}_{\mathrm{T}} / 3$ or equal to $\mathrm{N}_{\mathrm{T}} / 3$. The second part is an operation like conventional linear zero-force algorithm. The structure of the proposed hybrid QRDM and ZF detection algorithm is shown in Fig. 2(b).

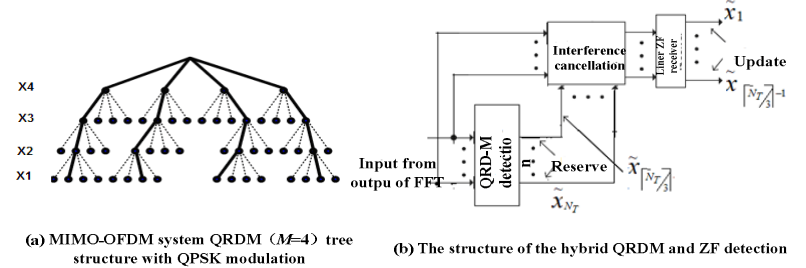

Figure 2. MIMO-OFDM system QRDM $(M=4)$ tree structure with QPSK modulation and the structure of the hybrid QRDM and ZF detection

The hybrid QRDM and ZF detection algorithm based on MIMO-OFDM system scheme is described as follows:

Step1: Denote set $A=\left\{1,2, \ldots, N_{T}\right\}$, set $B=\varnothing$.

For $i=1$ to $N$

$$
\begin{aligned}
& k=\underset{k \in A}{\arg \min }\left\|H_{(k)}\right\|^{2} ; \\
& \text { Hnew }_{(\mathrm{i})}=H_{(k)}, A=A \backslash k, B=B \cup k ;
\end{aligned}
$$$$
\text { End }
$$

Where $H_{(k)}$ represents the $k t h$ column of the matrix $H$ and Hnew is the channel matrix after sorting and its columns sorts as the gain of $H_{(k)}$ from minimum to maximum.

Step2: The conventional QRDM detection algorithm is firstly adopted. However, the algorithm should be stopped at $i=N_{T}-\left\lceil N_{T} / 3\right\rceil+1$.

Step3: Reserved the signals of the $N_{T}-\left\lceil N_{T} / 3\right\rceil+1$ detected layers and the signals can be denoted as $x^{*}=\left[\tilde{x}_{\left[N_{T} / 3\right]}, \ldots, \tilde{x}_{N_{T-1}}, \tilde{x}_{N_{T}}\right]^{T}$. It may cancel the interference of reserved signal to the other layers. Then a new MIMO-OFDM system with $\left(\left\lceil N_{T} / 3\right\rceil-1, N_{R}\right)$ antennas is obtained. For the new MIMO-OFDM system the signal relation between input and output is

$$
y_{\text {Update }}=y-H^{*} x^{*}
$$

Where $H^{*}=\left[\right.$ Hnew $\left._{\left\lceil N_{T} / 3\right\rceil}, \ldots, H n e w_{N_{T}}\right]$.

The channel matrix is update by

$$
H_{\text {Update }}=\left[\text { Hnew }_{1}, \ldots, \text { Hnew }_{\left\lceil N_{T} / 3\right\rceil-1}\right]
$$

And the filter matrix $G_{\text {Update }}$.

$$
G_{\text {Update }}=\left(H_{\text {Update }}^{{ }^{H}} H_{\text {Update }}\right)^{-1} H_{\text {Update }}{ }^{H}
$$

(12)

Step4: Use the conventional linear ZF detection. The other $\left\lceil N_{T} / 3\right\rceil-1$ signals are estimated by $x_{\text {others }}=G_{\text {Update }} y_{\text {Update }}$ $\left.=\left[\tilde{x}_{1}, \ldots, \tilde{x}\left[N_{T} / 3\right\rceil-1\right]\right]^{T}$ and all signals $\tilde{x}=\left[x_{\text {others }}^{T}, x^{* T}\right]^{T}$ are obtained.

Step5: Finally, sorting $x$ corresponding to the order in set $B$ and the output is the result of the hybrid QRDM$\mathrm{ZF}$ algorithm detection.

\section{B. Algorithm Analysis}

In the first part of the detection, the channel matrix $\mathrm{H}$ has been sorted as the column norm from minimum to maximum. The first detection signal is corresponding to the maximum column norm representing the maximum signal-to-noise (SNR) layer. As it's known, the bigger the SNR is, the more reliable of the detection signal is. By this way, the performance of the first detected signals has enhanced greatly. However, when it comes to the last few layers, the SNR is small. In order to lower the complexity of the algorithm, ZF receiver rather than QRDM receiver is used in the second part of the detection. Due to the performance of the first part $N_{T}-\left\lceil N_{T} / 3\right\rceil+1$ detected layers is the best in all layers, the performance of $\mathrm{ZF}$ detection will not influence the overall performance too much. Furthermore, when the $N_{T}-\left\lceil N_{T} / 3\right\rceil+1$ signals are reserved, its interference to other layers may be cancelled. The simulation results of this determination method will be given in Section V.

$$
\text { V. SIMULATION RESUlTS }
$$

In this section, computer simulations are presented to evaluate the performance of the proposed dynamic hybrid QRDM-ZF algorithm. The receiver detection is based on five paths Rayleigh fading environment without channel coding, assuming perfect channel estimation. For comparison purpose, $\mathrm{ZF}$ and QRDM receivers are also presented. In the simulation, the FFT size is 64 , and the cyclic prefix length $\mathrm{L}$ is 16 . The antenna configuration consists of 4 transmitter antennas, and 4 receiver antennas. The QPSK and 16-QAM modulation are used. Fig. 3 shows the comparison performance of three algorithms with different $M(M=1,2,3,4)$ by QPSK modulation and Fig. 4 explains the performance of those algorithms with $M(M=8,12,16)$ by $16 \mathrm{QAM}$ modulation.

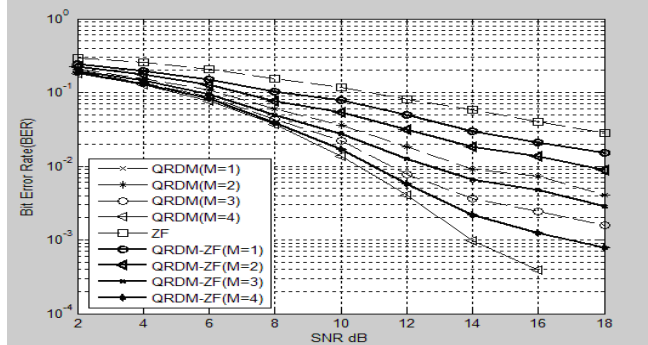

Figure.3 BER performance of ZF, QRDM and QRDM-ZF with M=1,2, 3, 4 by QPSK modulation in $4 \times 4$ MIMO-OFDM system without channel coding. 


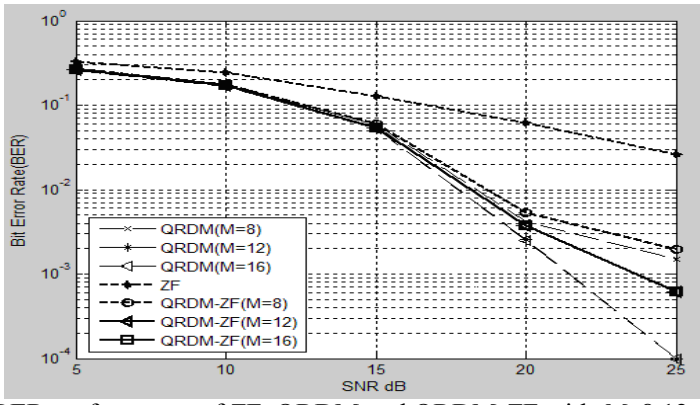

Figure.4 BER performance of ZF, QRDM and QRDM-ZF with $M=8,12,16$ by 16QAM modulation in $4 \times 4$ MIMO-OFDM system without channel coding.

\section{A. The Performance of Hybrid QRDM-ZF Algorithm Analysis}

From Fig.3 and Fig.4, it can be concluded that the performance of QRDM-ZF algorithm is better than that of ZF detection. However, even thought QRDM-ZF detection algorithm has lower complexity than QRDM algorithm, its performance is not much worse than QRDM algorithm. Compared with the conventional QRDM algorithm, the performance is about $2 \mathrm{~dB}$ degradation at $\mathrm{BER}=10^{-3}$ with 16QAM modulation and $2.1 \mathrm{~dB}$ degradation at $\mathrm{BER}=10^{-3}$ with QPSK modulation. The reason is that the channel matrix $\mathrm{H}$ has been sorted as the column norm from the minimum to the maximum. The performance of the first detected signals has enhanced greatly and it's reasonable to use ZF detection to the last few layers with small SNR.

B. Complexity Analysis

The branches that QRDM algorithm should search are defined as (13).

$$
L=S+\sum_{i=1}^{N_{T}-1} M S
$$

Where $\mathrm{L}$ is the total branches that should be searched, $\mathrm{S}$ and $\mathrm{M}$ are defined above in the QRDM receiver.

The effective branches of QRDM denote as $L_{Q R D M}$ and the effective branches of QRDM-ZF denote as $L_{Q R D M-Z F} . L_{Q R D M}$ and $L_{Q R D M-Z F}$ are defined in (14) and (15).

$$
\begin{aligned}
& L_{Q R D M}=S\left(M N_{T}-M+1\right) \\
& L_{Q R D M-Z F}=S\left(M N_{T}-M\left\lceil N_{T} / 3\right\rceil+1\right)
\end{aligned}
$$

Due to the less complexity of linear $\mathrm{ZF}$ detection, the complexity between QRDM and hybrid QRDM-ZF can be compared by effective searching branches. For 4 transmitter antennas and 4 receiver antennas, with QPSK and 16QAM modulation, the effective branches base on different $M$ are shown in Table I and Table II.

TABLE I.

DIFFERENT SEARCH BRANCHES OF QRDM AND QRDM-ZF BASED ON QPSK, $N_{R}=N_{T}=4$

\begin{tabular}{|c|c|c|c|c|}
\hline Value of $M$ & $L_{Q R D M}$ & $L_{Q R D M-Z F}$ & $\begin{array}{c}\text { Reduced } \\
\text { complexity }\end{array}$ & $\begin{array}{c}\text { Average reduced } \\
\text { complexity }\end{array}$ \\
\hline 1 & 16 & 12 & $25 \%$ & \multirow{2}{*}{$29.41 \%$} \\
\hline 2 & 28 & 20 & $28.57 \%$ & \\
\hline 3 & 40 & 28 & $30 \%$ & \\
\hline 4 & 52 & 36 & $30.77 \%$ & \\
\hline & \multicolumn{3}{|c|}{ TABLE II. } \\
\cline { 1 - 3 } & &
\end{tabular}

DIFFERENT SEARCH BRANCHES OF QRDM AND QRDM-ZF BASED

\begin{tabular}{|c|c|c|c|c|}
\hline 2 & 112 & 80 & $28.57 \%$ & \multirow{15}{*}{$32.08 \%$} \\
\hline 3 & 160 & 112 & $30 \%$ & \\
\hline 4 & 208 & 144 & $30.77 \%$ & \\
\hline 5 & 256 & 176 & $31.25 \%$ & \\
\hline 6 & 304 & 208 & $31.58 \%$ & \\
\hline 7 & 352 & 240 & $31.82 \%$ & \\
\hline 8 & 400 & 272 & $32 \%$ & \\
\hline 9 & 448 & 304 & $32.14 \%$ & \\
\hline 10 & 496 & 336 & $32.26 \%$ & \\
\hline 11 & 544 & 368 & $32.35 \%$ & \\
\hline 12 & 592 & 400 & $32.43 \%$ & \\
\hline 13 & 640 & 432 & $32.5 \%$ & \\
\hline 14 & 688 & 464 & $32.56 \%$ & \\
\hline 15 & 736 & 496 & $32.61 \%$ & \\
\hline 16 & 784 & 528 & $32.65 \%$ & \\
\hline
\end{tabular}
ON 16QAM, $N_{R}=N_{T}=4$

\begin{tabular}{|c|c|c|c|c|}
\hline $\begin{array}{c}\text { The value } \\
\text { of } \mathrm{M}\end{array}$ & $L_{Q R D M}$ & $L_{Q R D M-Z F}$ & $\begin{array}{c}\text { Reduced } \\
\text { complexity }\end{array}$ & $\begin{array}{c}\text { Average reduced } \\
\text { complexity }\end{array}$ \\
\hline 1 & 64 & 48 & $25 \%$ & \\
\hline
\end{tabular}

Form table I and table II, it can be concluded that the proposed scheme compared with QRDM algorithm is quite effective with a reduced complexity. In a $4 \times 4$ MIMO-OFDM system by QPSK modulation, compared with QRDM algorithm, the proposed technique has decreased the complexity by $29.41 \%$ on average. In the same systems by 16 QAM modulation, the hybrid QRDM-ZF algorithm has decreased the complexity by $32.08 \%$ on average.

\section{CONCLUSIONS}

In this paper, a new efficiency algorithm for MIMOOFDM system was proposed. It enhances the detection performance by using the QRD-M and reduces the complexity by applying the conventional ZF detection algorithm. Simulation results show that if the complexity of the detection algorithm is reduced, the detection performance will decrease. In fact, a signal detection algorithm with a low complexity and high BER performance is a troublesome subject. The traditional QRD-M is difficult because of its high complexity. Therefore, the proposed hybrid QRDM-ZF detection can be used for a MIMO-OFDM receiver requiring not very high performance but needing very low complexity.

\section{REFERENCES}

[1] P. W. Wolniansky, G. J. Foschini, G. D. Golden, and R. A. Valenzuela,

"V-BLAST: an architecture for achieving very high data rate over rich scattering wireless channels," in Proc. ISSSE'98, pp. 295-300, Sept.1998.

[2] R. Bohnke, D. Wudden, and K. Kammeyer, "Reduced complexity MMSE detection for BLAST architectures," in Proc. GLOBECOM 2003, pp. 2258-2262, Sept. 2003.

[3] K. J. Kim and R. A. Iltis, "Joint detection and channel estimation algorithms for QS-CDMA signals over time-varying Channels," IEEE Trans. Commun., vol. 50, pp. 845-855, May 2002.

[4] K. J. Kim, J. Yue, R. A. Iltis, and J. D. Gibson, "A QRD-M/Kalman filter-based detection and channel estimation algorithm for MIMOOFDM systems," IEEE Trans. Wireless Commun., vol. 4, no. 2, pp. 710-721, Mar. 2005.

[5] B. Hassibi., "An Efficient Square-Root Algorithm for blast," in Proc. IEEE Intl. Conf. Acoustic, Speech, Signal Processing, June,2000, Istanbul, Turkey, pp.5-9

[6] Van Z. A. Space Division Multiplexing Algorithm [C]. Electrotechnical Conference. MELECON 2000, 2000, vol. 3: 1218-1221.

[7] Li Wei Cheng Shi-xin Chen Ming,"A Improved QRD—M Algorithm for MIMO Signal Detection", Journal of Electronics \&Information Technology, Aug.2008, Vol,30, No.8.

[8] Myung-Sun Baek, Young-Hwan You, and Hyoung-Kyu Song,"Combined QRD-M and DFE Detection Technique for Simple and Efficient Signal Detection in MIMO-OFDM Systems", IEEE Trans. Wireless Commun., vol. 8, no. 4, April 2009. 